\title{
Case report: Whole exome sequencing of circulating cell-free tumor DNA in a follicular thyroid carcinoma patient with lung and bone metastases
}

Journal of Circulating Biomarkers Volume 7: I-6 (C) The Author(s) 2018 Reprints and permissions: sagepub.co.uk/journalsPermissions.nav DOI: $10.1177 / 1849454418763725$ journals.sagepub.com/home/cbx (9)AGE

\author{
Jianlu Song and Zhili Yang
}

\begin{abstract}
Metastatic follicular thyroid carcinoma (FTC), unresectable or resistance to radioactive iodine, is associated with poor survival. It is believed that this kind of FTC is driven by mutated genes. However, what kind of changes of genome and underlying mechanisms are elusive. The aim of this article is to understand whether there are somatic mutations in circulating cell-free tumor DNA (cfDNA) in a FTC patient with lung and bone metastases. A 55-year-old woman was diagnosed with FTC with bone and lung metastases. Appropriate amounts of DNA were extracted from formalin-fixed, paraffin-embedded thyroid tumor, peripheral cell-free plasma, and peripheral blood leukocytes and then sequenced. The significance of DNA sequencing was evaluated. There were 13,519 common variants in both tissue DNA and cfDNA. Fifty-five somatic mutations were identified in tumor, with 5 of them nonsynonymous. Seventy-two somatic mutations were found in cfDNA, with 2 of them causing amino acid change. Sixteen common alterations existed in both samples, that is, $31.3 \%$ of all the tissue somatic mutations. This pilot study provided proof that cfDNA represents the genomic characteristics of FTC primary tissue DNA well, but also metastatic tumors. Further studies are needed to better prove the effectiveness of cfDNA in the field of thyroid cancer metastatic mechanism research and real-time monitoring.
\end{abstract}

\section{Keywords}

Follicular thyroid carcinoma, metastasis, circulating cell-free DNA, whole exome sequencing

Date received: II December 2017; accepted: 2 February 2018

\section{Introduction}

Most follicular thyroid carcinomas (FTCs) grow slowly after they are first identified at an early stage. It is frequently cured with adequate surgical management and radioactive iodine ablation therapy. However, few cases develop fast with lung and bone metastases. Most metastatic FTCs are unresectable and refractory to radioactive iodine and associated with poor survival. ${ }^{1}$ Analysis of carcinogenesis has revealed that gene mutations play a very important role in the progression and development of tumors. The genetic profile of solid tumors is often obtained from surgical or biopsy specimens. However, tumor samples may not be obtained due to the confirmation of metastasis. In that situation, blood samples or biopsy can be used for analysis. Several studies of circulating cell-free DNA (cfDNA) in plasma have been used for analyzing individual loci, genes, or structural variants to quantify tumor burden and detect previously characterized resistance-conferring mutations. ${ }^{2-4}$ Individual mutations of cfDNA and SLC5A8 and SLC26A4 hypermethylation

Department of General Surgery, Shanghai Jiao Tong University Affiliated Sixth People's Hospital, Shanghai, China

\section{Corresponding Author:}

Zhili Yang, Department of General Surgery, Shanghai Jiao Tong University Affiliated Sixth People's Hospital, 600 Yi-Shan Road, Shanghai 200233, China.

Email: yangzhililaoshi@126.com 


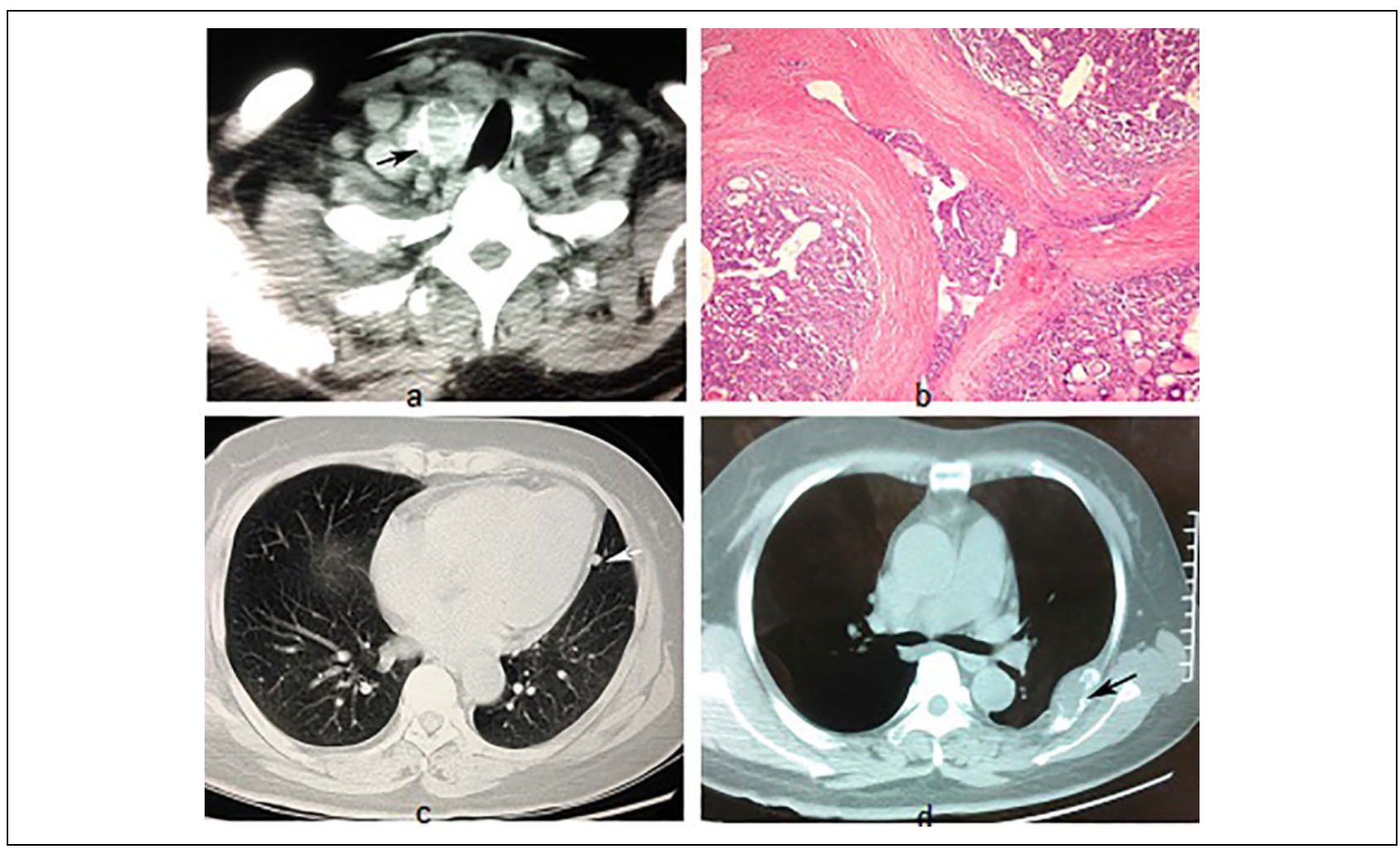

Figure I. Imaging of primary and metastasis lesions and pathological examination of primary thyroid lesion. (a) Imaging CT of primary thyroid lesion. Black arrow indicates tumor location. (b) Histological analysis of primary lesion of FTC ( $\times 100)$. (c) Lung metastasis as indicated by white arrow. (d) Rib metastasis as indicated by black arrow. CT: computed tomography; FTC: follicular thyroid carcinoma.

of thyroid carcinoma including follicular carcinoma have been reported. ${ }^{5}$ Given that sequencing of entire genes to detect FTC mutations in circulating DNA has not been demonstrated, we report a somatic mutational analysis of cfDNA in a FTC patient with lung and bone metastases by whole exome sequencing (WES).

The results demonstrate that the detection of cfDNA may reflect the mutation of FTC and further develop to be a better way for monitoring the progression of thyroid cancer.

\section{Patient and methods}

\section{Patient}

A 55-year-old woman was diagnosed with FTC with synchronous bone and lung metastases and then performed total thyroidectomy at Shanghai Jiao Tong University Affiliated Sixth People's Hospital (Figure 1). The informed consent for DNA sequencing was obtained from the patient. Preoperative peripheral cell-free plasma and peripheral blood leukocytes and postoperative formalinfixed, paraffin-embedded (FFPE) thyroid tumor of this patient were obtained. Procedure and protocol were reviewed and approved by the ethics committee of the Sixth People's Hospital.

\section{DNA extraction}

Three different types of DNA were obtained from this present patient, including peripheral blood leukocytes, FFPE thyroid tumor tissue, and cfDNA. Total amount of DNA were extracted according to the manufacturer's instructions using QIAamp DNA Blood Mini Kit (cat\# 51106), QIAamp DNA FFPE Tissue Kit (cat\# 56404), and cfDNA QIAamp circulating Nucleic Acid Kit (cat\# 5514), respectively. DNA concentrations were $81 \mathrm{ng} / \mu \mathrm{l}, 85 \mathrm{ng} / \mu \mathrm{l}$, and $81 \mathrm{ng} / \mu \mathrm{l}$, respectively, and the optical density $260 / 280$ is $1.86,1.87$, and 1.89 , respectively. DNA samples were then subjected to Agilent 2100 for more accurate tests. All three DNA samples passed a strict quality test.

\section{Whole exome sequencing}

The three different samples above were proceeded with WES. Briefly, first, DNA libraries were established with Agilent SureSelect Human All ExonV5 kits, covering about 23,000 genes. After the quality test, qualified libraries were sequenced as 100 -bp paired-end reads on Illumina HiSeq 2000 platform (Illumina, San Diego, California, USA) according to the manufacturer's instruction. All of the experiments were carried out in Zhangjiang Center for Translational Medicine, Shanghai, China. 
Table I. Outline of the WES of cfDNA, tumor DNA, and peripheral blood DNA.

\begin{tabular}{lccc}
\hline & $\begin{array}{c}\text { Peripheral } \\
\text { blood DNA }\end{array}$ & $\begin{array}{c}\text { Tumor } \\
\text { DNA }\end{array}$ & cfDNA \\
\hline Total effective sequence data $(\mathrm{Gb})$ & 11.0 & 10.0 & 11.2 \\
Coverage rate on targeted region & 99.76 & 99.67 & 99.8 \\
Mean depth & 140.04 & 139.59 & 133.21 \\
No. of SNVs & 41,410 & 41,874 & 41,206 \\
No. of Indels & 3259 & 3490 & 3486 \\
\hline
\end{tabular}

cfDNA: cell-free DNA; WES: whole exome sequencing.

\section{Data analysis}

Clean data were achieved using FastQC and low quality reads were filtered. Burrows-Wheeler Alignment (0.7.12) methods were adopted to map the clean reads to reference genome (UCSC hg19). Then, Picard (http://picard.source forge.net/) and Genome Analysis Toolkit (GATK) methods were used for duplicate removal, local realignment, and base quality recalibration. GATK unified genotyper was used for variants calling.

ANNOVAR (2015-03-22) software was used to annotate variants for function (exonic, TR, intronic) reference gene, exonic function (synonymous, nonsynonymous, frameshift, stopgain, unknown), amino acid change, allele frequency (AF) 1000 Genomes Project, and dbSNP reference number.

Somatic mutations were analyzed by subtracting the variants of the peripheral blood DNA from the tumor DNA or cfDNA. $T$-test was used to compare the AF between different types of samples. Pearson correlation coefficient was adopted to calculate the relationship between different types of samples.

\section{Results}

\section{Exome sequencing, sequence alignment, and variant calling}

In this study, we totally sequenced three different types of DNAs from one patient. More than $10 \mathrm{~Gb}$ sequencing data were generated per sample. The average sequencing depth for peripheral blood DNA, tissue DNA, and cfDNA is $139 \times, 140 \times$, and $133 \times$, respectively. For three samples, more than $98.7 \%$ of the exome was covered at least $10 \times$. The coverage rate for the above three samples was larger than $99.6 \%$. More details are shown in Table 1. The mutation types in different samples were nearly the same.

\section{Variations identified in different samples}

We compared variations (both SNV and Indel) in different DNA samples. Generally, a large concordance between the three types of samples was observed (Figure 2(a)). There were 13,519 common variants in both tissue DNA and cfDNA. They shared more than $98.6 \%$ common variants compared with peripheral blood sample. The coefficient rate was 0.95 ( $p$ value $\left.<10^{-15}\right)$ and 0.96 ( $p$ value $<$ $10^{-15}$ ), respectively; $1.39 \%$ of variants identified in tissue DNA were not found in peripheral blood and $1.13 \%$ of variants identified in cfDNA did not exist in peripheral blood. Comparing tissue DNA with cfDNA showed that 99.2\% variants were identical (Figure 2(b) to (d)). The Pearson coefficient between cfDNA and tissue DNA is 0.95 (Figure 2(e)).

Somatic mutations were determined by subtracting the variants of the peripheral blood leukocytes DNA from the tumor DNA or cfDNA. Fifty-five somatic mutations were found in tissue DNA, in which 5 mutations were nonsynonymous. Seventy-two somatic mutations were found in cfDNA, in which 2 of them changed amino acid. Sixteen common alterations existed in both samples, that is, $31.3 \%$ in all the tissue somatic mutations (Table 2). Most of the common mutations were synonymous. In cfDNA, only GLUD2 (c.G103A: p.G35R) and HLA-B (c.A652G: p.I218 V) were nonsynonymous mutations, of which GLUD2 (c.G103A: p.G35R) was also found in the tumor DNA.

\section{Discussion}

Genome-wide sequencing of plasma DNA was first used in prenatal diagnostics, demonstrating comprehensive genome characteristics. ${ }^{6}$ Recent studies showed that sequencing of circulating cfDNA from plasma is a potential tool for monitoring advanced cancer. Compared with traditional biopsy, cfDNA sequencing is noninvasive, easy to get samples repeatedly, and to large extent reflects the comprehensive genomic characteristic of the tumor progress. ${ }^{2-4,7}$ In our data, cfDNA from FTC was for the first time sequenced together with primary tumor in the advanced thyroid cancer. The results demonstrate that SNV and mutation rate of cfDNA are characterized by the features of FTC, and both tissue DNA and cfDNA shared more than $95 \%$ common alterations. It will be helpful for doctors or researchers to use cfDNA as a new method for the diagnosis and monitoring of thyroid carcinoma.

Recent studies showed that cancers arise through a process of somatic evolution that can result in substantial subclonal heterogeneity within tumors, and a large fraction of polyclonal tumors and a larger sub-clonal mutation fraction may be associated with relapse and metastasis. ${ }^{8,9}$ It has been established that there were different mutation variants between primary and metastatic tumors. In our results, there were different variants identified in tissue DNA and cfDNA except for common alterations. One of the reasons may be the existence of distinct mutational genes of bone and lung metastatic tumors in cfDNA. ${ }^{7}$ In addition, the detection of genomic alteration by sequencing of cfDNA is to some extent dependent on the AF of the mutant alleles in the tissue DNA. AF value of variants which existed in both cfDNA and tissue DNA is significantly larger than that of variants only 


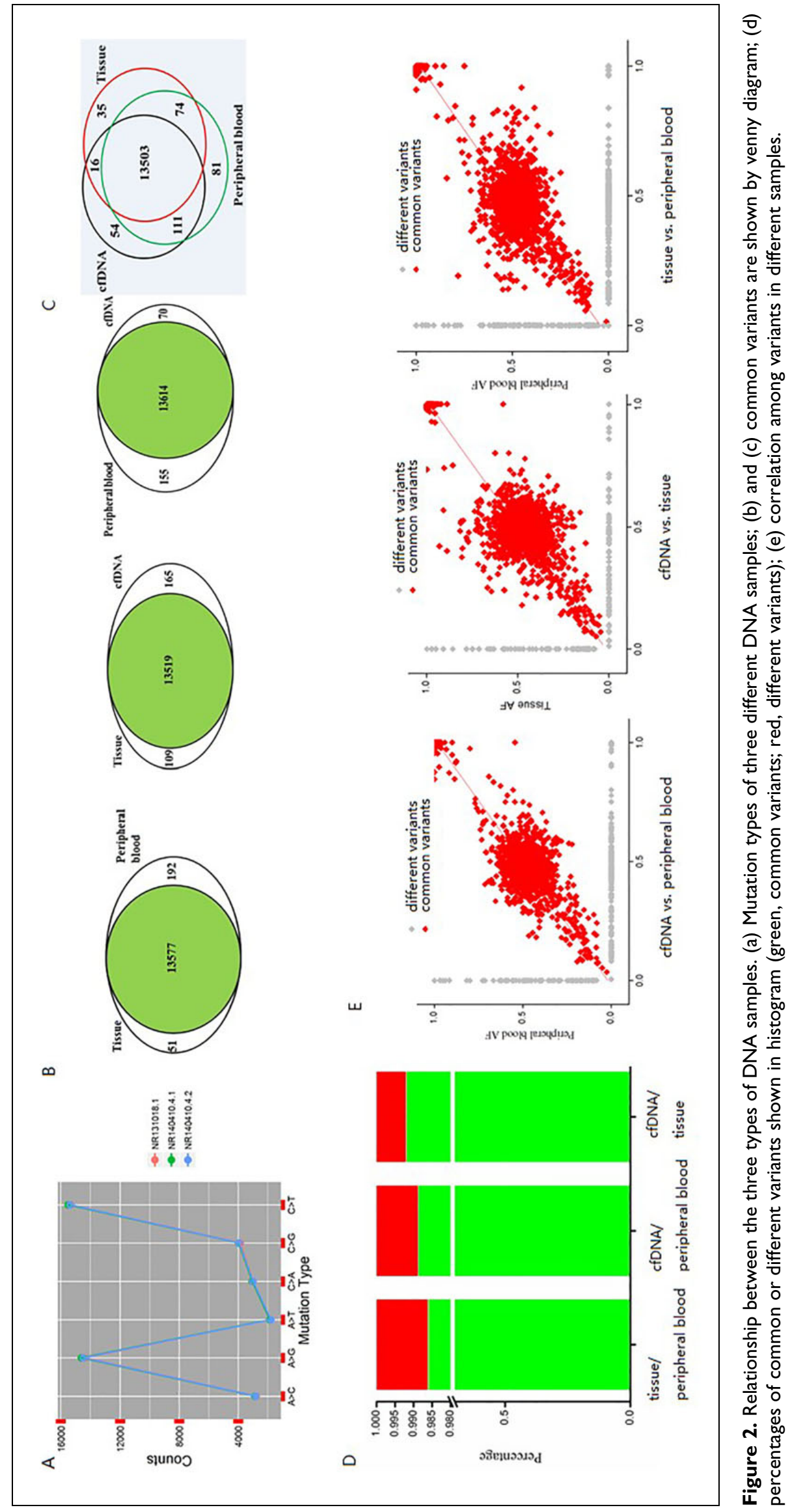


Table 2. List of genes with somatic mutations in tumor and cfDNA.

\begin{tabular}{|c|c|c|c|c|}
\hline \multicolumn{2}{|c|}{ Tissue somatic mutation genes } & \multicolumn{2}{|c|}{ cfDNA somatic mutation genes } & \multirow{2}{*}{$\begin{array}{l}\text { Intersection (31\%) } \\
\text { FAM69A }\end{array}$} \\
\hline FAM69A & UBE2L3 & TTC39A & CILP2 & \\
\hline RPL5 & TFAP2E & FAM69A & ZNF43I & RPL5 \\
\hline ANKRD45 & $\mathrm{FBXO} 28$ & RPL5 & DEFBI 24 & ANKRD45 \\
\hline SLC9A2 & RHOA & TMEM56 & LOCI0I92763I & RAPGEF4-ASI \\
\hline RAPGEF4-ASI & LOCIOI 243545 & ANKRD45 & UBE2L3 & EOMES \\
\hline ATF2 & TAPTI-ASI & NUCKSI & FAM9B & TWF2 \\
\hline EOMES & BRD9 & DIEXF & SH3KBPI & NCALD \\
\hline TWF2 & NEUROD6 & CNRIPI & EFNBI & MPVI7L \\
\hline RUVBLI & TMEM229A & ERMN & PJAI & UBE2L3 \\
\hline TM4SFI9-ASI & Unc5D & RAPGEF4-ASI & MOSPDI & Unc5D \\
\hline MARCH6 & DPYI9L4 & EOMES & MIR4424 & FJXI \\
\hline KCTD20 & GLIPR2 & TWF2 & MAP6DI & SNX20 \\
\hline TMEM248 & HRAS & GOLIM4 & HLA-B & CDRTI5L2 \\
\hline DNAJC2 & FJXI & HESI & HLA-DRBI & LOCI00287072 \\
\hline NCALD & FAM86Cl & PDCL2 & PPIA & EID2 \\
\hline HAS2-ASI & RCORI & ANXA5 & NCFIC & GLUD2 \\
\hline RABIB & PDCD7 & CPE & Unc5D & \\
\hline CXCR5 & CHRNA5 & NUDCD2 & FAM35BP & \\
\hline LNX2 & SNX20 & CASCI5 & FAM35DP & \\
\hline CTAGEIOP & ALOXI5PI & EPB4IL2 & $\mathrm{FJXI}$ & \\
\hline EMC7 & CDRTI5L2 & FZDI & LOC338797 & \\
\hline SNN & LOCI00287072 & XPO7 & SLITRKI & \\
\hline MPVI7L & VATI & NCALD & MIR45II & \\
\hline DCXR & EID2 & MSANTD3-TMEFFI & IDH3A & \\
\hline RWDD2B & NCOA3 & TMEFFI & GNPTG & \\
\hline \multirow[t]{10}{*}{ GLUD2 } & & CSGALNACT2 & SNX20 & \\
\hline & & AMBRAI & PAFAHIBI & \\
\hline & & NAALAD2 & CDRTI5L2 & \\
\hline & & FGFRIOP2 & LOCI00287072 & \\
\hline & & MICU2 & CERSI & \\
\hline & & MEIS2 & GDFI & \\
\hline & & MPVI7L & EID2 & \\
\hline & & ZNF77I & GNG8 & \\
\hline & & TOMIL2 & ZNF8I6-ZNF32IP & \\
\hline & & SMARCEI & GLUD2 & \\
\hline
\end{tabular}

cfDNA: cell-free DNA.

existed in tissue DNA, indicating that the higher the AF is in tissue DNA, the easier the cfDNA is to be detected. Thirdly, the sensitivity of cfDNA sequencing was dependent on sequencing depth. ${ }^{10}$ High sequencing depth will help to find rare mutations in tissue DNA. In this study, we acquired 100 sequencing depth, so the mutation with less than $1 \% \mathrm{AF}$ is unable to be identified theoretically.

Except for FAM69A, RAPGEF4-AS1, LOC100287072, and CDRT15L2, most of these common mutation genes are related to many functions of cancer cells, and Unc5D gene inhibits thyroid cancer cell behaviors. ${ }^{11}$ As a common nonsynonymous mutant gene GLUD2, its activity supports cancer cell proliferation under glutamine depletion. ${ }^{12}$ Therefore, these gene mutations may be involved in the metastatic process of FTC.

In summary, we first performed WES from cfDNA of an advanced thyroid cancer patient. This pilot study provided preliminary evidence that cfDNA represents the genomic characteristics of primary FTC, implying the risk of metastasis. Further studies guarantee sequencing of metastatic tumors, increase more cases, and determine the biomarker of cfDNA for metastasis of thyroid cancer.

\section{Acknowledgements}

The authors thank Dr Bo Peng and Dr Yanbing Qi (Zhangjiang Center for Translational Medicine, Shanghai, China) for sequencing analysis.

\section{Declaration of Conflicting Interests}

The author(s) declared no potential conflicts of interest with respect to the research, authorship, and/or publication of this article.

\section{Funding}

The author(s) disclosed receipt of the following financial support for the research, authorship, and/or publication of this article: This work was supported by the National Natural Science Foundation of China (\# 81472499). 


\section{References}

1. Kapiteijn E, Schneider TC, Morreau H, et al. New treatment modalities in advanced thyroid cancer. Ann Oncol 2012; 23(1): 10-18.

2. Murtaza M, Dawson SJ, Tsui DW, et al. Non-invasive analysis of acquired resistance to cancer therapy by sequencing of plasma DNA. Nature 2013; 497(7447): 108-112.

3. Forshew T, Murtaza M, Parkinson C, et al. Noninvasive identification and monitoring of cancer mutations by targeted deep sequencing of plasma DNA. Sci Transl Med 2012; 4(136): 136ra68.

4. Bettegowda C, Sausen M, Leary RJ, et al. Detection of circulating tumor DNA in early- and late-stage human malignancies. Sci Transl Med 2014; 6(224): 224ra24.

5. Zane M, Agostini M, Enzo MV, et al. Circulating cellfree DNA, SLC5A8 and SLC26A4 hypermethylation, BRAF(V600E): a non-invasive tool panel for early detection of thyroid cancer. Biomed Pharmacother 2013; 67(8): 723-730.

6. Vlkova B, Szemes T, Minarik G, et al. Advances in the research of fetal DNA in maternal plasma for noninvasive prenatal diagnostics. Med Sci Monit 2010; 16(4): RA85-RA91.

7. Crowley E, Di Nicolantonio F, Loupakis F, et al. Liquid biopsy: monitoring cancer-genetics in the blood. Nat Rev Clin Oncol 2013; 10(8): 472-484.

8. Marusyk A, Tabassum DP, Altrock PM, et al. Non-cellautonomous driving of tumour growth supports sub-clonal heterogeneity. Nature 2014; 514(7520): 54-58.

9. Zhang J, Fujimoto J, Zhang J, et al. Intratumor heterogeneity in localized lung adenocarcinomas delineated by multiregion sequencing. Science 2014; 346(6206): 256-259.

10. Klevebring D, Neiman M, Sundling S, et al. Evaluation of exome sequencing to estimate tumor burden in plasma. PLoS One 2014; 9(8): e104417.

11. Zhang MM, Sun F, Cui B, et al. Tumor-suppressive function of UNC5D in papillary thyroid cancer. Oncotarget 2017; 8(56): 96126-96138.

12. Takeuchi Y, Nakayama Y, Fukusaki E, et al. Glutamate production from ammonia via glutamate dehydrogenase 2 activity supports cancer cell proliferation under glutamine depletion. Biochem Biophys Res Commun 2018; 495(1): 761-767. 